\title{
Gene loops function to maintain transcriptional memory through interaction with the nuclear pore complex
}

\author{
Sue Mei Tan-Wong, ${ }^{1}$ Hashanthi D. Wijayatilake, ${ }^{1}$ and Nick J. Proudfoot ${ }^{2}$ \\ Sir William Dunn School of Pathology, University of Oxford, Oxford OX1 3RE, United Kingdom
}

\begin{abstract}
Inducible genes in yeast retain a "memory" of recent transcriptional activity during periods of short-term repression, allowing them to be reactivated faster when reinduced. This confers a rapid and versatile gene expression response to the environment. We demonstrate that this memory mechanism is associated with gene loop interactions between the promoter and $3^{\prime}$ end of the responsive genes HXK1 and GAL1::FMP27. The maintenance of these memory gene loops (MGLs) during intervening periods of transcriptional repression is required for faster RNA polymerase II (Pol II) recruitment to the genes upon reinduction, thereby facilitating faster mRNA accumulation. Notably, a sua7-1 mutant or the endogenous INO1 gene that lacks this MGL does not display such faster reinduction. Furthermore, these MGLs interact with the nuclear pore complex through association with myosin-like protein 1 (Mlp1). An mlp1s strain does not maintain MGLs, and concomitantly loses transcriptional memory. We predict that gene loop conformations enhance gene expression by facilitating rapid transcriptional response to changing environmental conditions.
\end{abstract}

[Keywords: RNA polymerase II transcription; gene loops; transcriptional memory; S. cerevisiae; nuclear pore complex]

Supplemental material is available at http://www.genesdev.org.

Received May 21, 2009; revised version accepted September 21, 2009.

Studies of the mechanism of gene activation have long focused on the process of promoter selection. However, we and others demonstrated that once transcription is initiated, the promoter and 3 ' end of the gene immediately interact, resulting in a looped gene conformation (O'Sullivan et al. 2004). These intragenic loops are transcription-dependent and have been observed in varied Saccharomyces cerevisiae genes, in Schizosaccharomyces pombe (M Gullerova and NJ Proudfoot, unpubl.), in human genes such as BRCA1, and even in proviral HIV-1 integrants (Ansari and Hampsey 2005; Singh and Hampsey 2007; Perkins et al. 2008; Tan-Wong et al. 2008). Formation of these promoter-3'-end gene loops are dependent on transcription factors such as the yeast TFIIB homolog Sua7 (Singh and Hampsey 2007) and Kin28 (O'Sullivan et al. 2004), mRNA processing factors such as Ssu72 and Ptal (Ansari and Hampsey 2005), and also cis-acting mRNA-processing elements such as the polyadenylation and splicing signals (Perkins et al. 2008). While widespread, the function of these loop structures has remained enigmatic. We now demonstrate a direct

\footnotetext{
${ }^{1}$ These authors contributed equally to this work.

${ }^{2}$ Corresponding author.

E-MAIL Nicholas.proudfoot@path.ox.ac.uk; FAX 44-1865-275556.

Article is online at http://www.genesdev.org/cgi/doi/10.1101/gad.1823209.

Freely available online through the Genes \& Development Open Access option.
}

role for gene loops by showing their association with transcriptional memory in S. cerevisiae.

Transcriptional memory refers to a gene "remembering" its previous transcriptionally active state. Such memory confers the ability of a faster rate of transcription initiation and thus faster gene expression when reinduced following a short intervening period of repression. Transcriptional memory has been described for the highly expressed inducible $S$. cerevisiae genes GAL1 and GAL7 (Brickner et al. 2007; Kundu et al. 2007; Zacharioudakis et al. 2007). A variety of molecular factors have been associated with this phenomenon. At the chromatin level, factors such as the H2A.Z histone variant (Brickner et al. 2007) and the chromatin remodeling enzyme SWI/SNF (Kundu et al. 2007) have been implicated in transcriptional memory, whereas a cytoplasmic determinant, the yeast catabolic enzyme Gall, was also shown to be sufficient to confer memory (Zacharioudakis et al. 2007).

Significantly, GAL genes and INO1, as well as other inducible genes such as HXK1, HSP104, SUC2, and $\alpha$-factorinduced genes, are found to relocate to the nuclear periphery and associate with the nuclear pore complex (NPC) upon activation (Brickner and Walter 2004; Casolari et al. 2004, 2005; Cabal et al. 2006; Dieppois et al. 2006; Drubin et al. 2006; Schmid et al. 2006; Taddei et al. 2006; Brickner et al. 2007; Sarma et al. 2007). Such nuclear peripheral localization is thought to promote efficient and maximal 
transcriptional activation (Brickner and Walter 2004; Taddei et al. 2006; Brickner et al. 2007). INO1 and GAL1 also remain at the nuclear periphery for some period after glucose repression, and for GAL1, this NPC localization is thought to help its rapid reinduction memory (Abruzzi et al. 2006; Brickner et al. 2007; Chekanova et al. 2008; Vodala et al. 2008).

One of the NPC proteins potentially involved in these processes is myosin-like protein 1 (Mlp1), a $218-\mathrm{kDa}$ coiled-coil protein located among the intranuclear filaments of the NPC basket. Mlp1 is thought to be involved in the quality control of mRNA prior to cytoplasmic export, as it is required for the nuclear retention of nonspliced, intron-containing mRNAs (Galy et al. 2004; Vinciguerra et al. 2005). Mlp1 is, however, also seen to have many diverse functions, and has been implicated in mediating active gene interactions at the nuclear pore. Among such interactions is Mlp1's association with $\alpha$-factor-induced genes (Casolari et al. 2005), its enriched binding to active glycolytic genes (Casolari et al. 2004), and its interaction with actively transcribing GAL promoter regions in a SAGA complex-dependent manner (Luthra et al. 2007).

The mechanism of how NPC localization enhances gene expression by conferring rapid reinduction memory is still debated. Our results demonstrate that transcription-dependent gene loop structures in HXK1 and GAL1::FMP27 are maintained for up to a 1 -h period of intervening repression. These maintained loop structures are termed memory gene loops (MGLs). Cells reinduced within this 1-h period display faster RNA polymerase II ( $\mathrm{Pol}$ II) recruitment and thereby faster transcription initiation; a reinduction "memory." Maintenance of the MGLs is dependent on Mlp1. Consistent with this, Mlp1 displays a 5'/3' bimodal distribution on HXK1 at time points coincident with MGL presence, but not during their absence. mlp1s mutants disrupt HXK1 tethering to the NPC and lose MGL maintenance during intervening repression, with concomitant loss of transcriptional memory. Furthermore, we show that INO1, a gene naturally lacking the maintenance of MGLs during repression, shows no transcriptional memory. Similarly, a sua 7-1 mutation that disrupts gene looping while keeping the NPC intact causes a loss of transcriptional memory. We propose that NPC-tethered MGL structures are maintained during intervening periods of repression, thereby holding genes in an active conformation and so facilitating faster reinduction.

\section{Results}

The expression of many yeast genes is regulated by growth in differing nutrient conditions. Commonly studied genes are those of the GAL cluster that code for enzymes involved in the catalysis of galactose, and HXK1, which codes for Hexokinase isoenzyme 1, a protein that catalyzes phosphorylation of glucose during glucose metabolism. The expression of these genes is regulated by growth in different carbon sources; transcription is repressed in the presence of glucose, and is induced when galactose is used as the primary carbon source.
Defective Pol II regulation and release during HXK1 repression in mlp1s

We initially investigated the kinetics of transcription during induction and repression of these environmentally responsive genes. We show that HXK1 mRNA levels respond as expected to galactose induction and glucose repression (Fig. 1B), the kinetics consistent with previous studies (Herrero et al. 1995). We further analyzed the kinetics of HXK1 repression following transfer from galactose to glucose, and monitored the transcriptional activity of HXK1 by determining Pol II occupancy across the gene using chromatin immunoprecipitation (ChIP) (Fig. 1C). Consistent with other studies (Mason and Struhl 2005), we show that within $30 \mathrm{sec}$ of glucose inhibition, the Pol II ChIP signal disappears from the promoter (probe A), indicative of immediate cessation of Pol II initiation at the promoter, and a concomitant reduction is observed over the further $3^{\prime}$-positioned probes. Longer time points show a rapid and directional loss of Pol II extending across the gene, consistent with already elongating polymerases completing their final wave of $5^{\prime}$-to-3' transcription before disengaging from the gene. Pol II is almost completely absent from the gene by $5-8 \mathrm{~min}$.

Parallel ChIP analysis was performed on a strain lacking Mlp1, the NPC-associated nucleoplasmic protein implicated in mediating active gene interactions with the NPC (Casolari et al. 2005; Luthra et al. 2007). Interestingly, Pol II release from the downstream region of the gene was initially slower in an $\mathrm{mlp} 1 \Delta$ strain following glucose repression (Fig. 1C; cf. probe $\mathrm{C}$ and D signals between wild type and mlp1A). However, mRNA analysis of the mlp1s strain in a time course similar to that in Figure 1B did not show a significant difference in mRNA levels in comparison with wild type during first-round induction/repression (Supplemental Fig. 1A). This indicated a possible defect in the regulation of transcriptional machinery following HXK1 repression in mlp1s, the effects of which may be observed only in subsequent rounds of reinduction.

\section{Mlp1 is required for maintaining gene loops during repression}

As Mlp1 is implicated in mediating active gene interactions with the NPC (Casolari et al. 2005; Luthra et al. 2007), we investigated the possible involvement of Mlp1 in loop interactions of $H X K 1$, a gene known to localize to the NPC upon activation (Taddei et al. 2006). Chromosome conformation capture (3C) analysis was performed using a range of $3 \mathrm{C}$ primers across HXK1 (Supplemental Fig. 2A), and a clear intragenic loop was detected between the $5^{\prime}$ upstream activating sequence (UAS) and the $3^{\prime}$ end during active transcription (Fig. $1 \mathrm{D}$, time point $0 \mathrm{~min}$ ). This HXK1 loop profile is consistent with all other budding yeast, fission yeast, and mammalian gene loops observed thus far, and provides a further example of the universal nature of gene loops.

Gene loops have thus far been shown to be transcription-dependent, as they are absent in nontranscribing 
Tan-Wong et al.

(A)

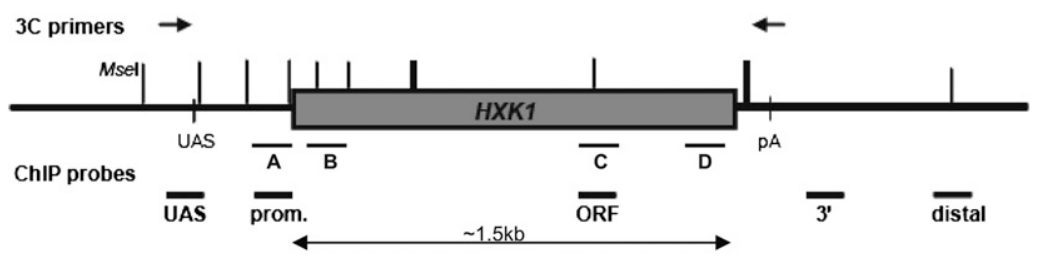

(B)

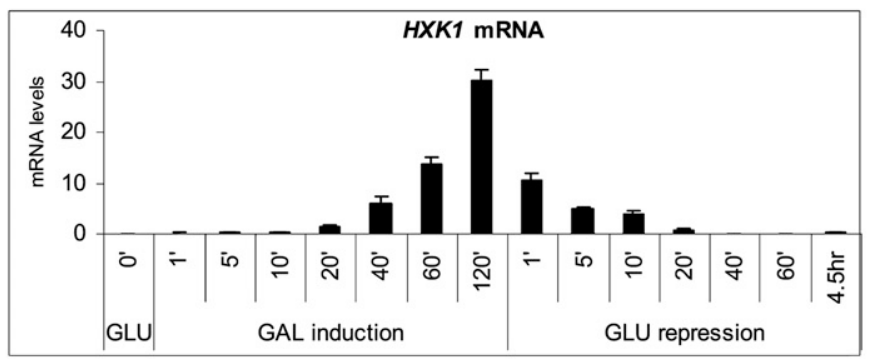

(C)

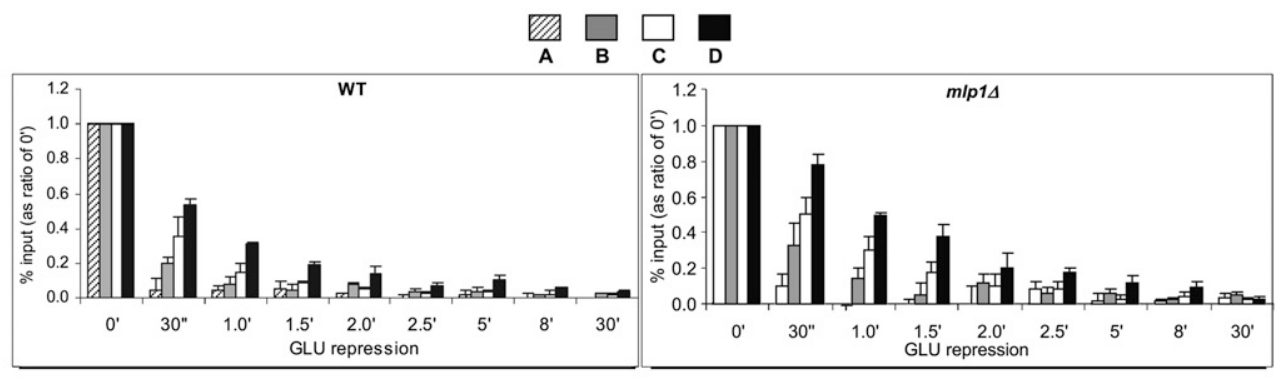

(D)
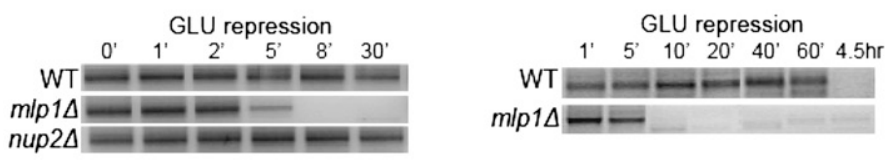

(E)
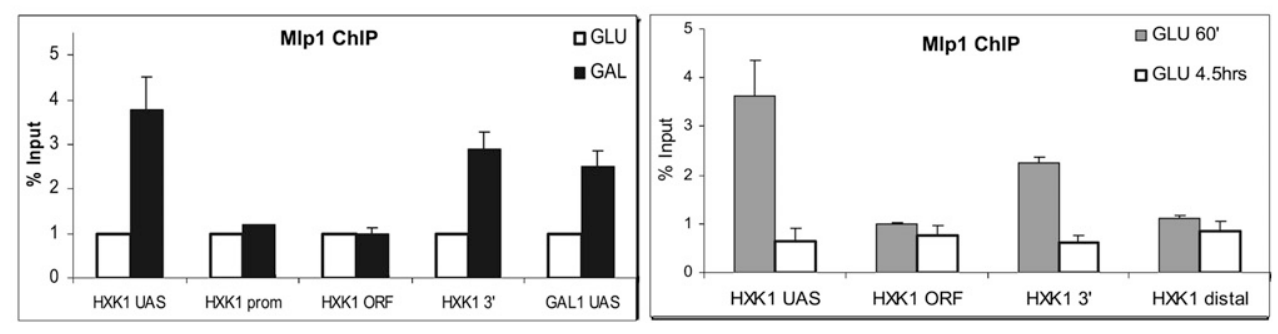

Figure 1. Mlp1 is required for gene loop maintenance during glucose shutdown. (A) Schematic of $H X K 1$ indicating positions of $3 \mathrm{C}$ primers (above) and ChIP probes (below). Vertical lines depict MseI restriction sites used in 3C analysis (thicker lines depict very closely spaced restriction sites). (B) RT-qPCR analysis of $H X K 1 \mathrm{mRNA}$ kinetic response to galactose induction and glucose repression. (C) Pol II ChIP analysis on $H X K 1$ in wild type and $m l p 1 \Delta$ during a glucose shutdown time course. A-D refer to positions along the gene as indicated in $A$. Values are shown as a ratio of time point $0 \mathrm{~min}^{\prime}$. (D) HXK1 time-course $3 \mathrm{C}$ analysis on wild-type, mlp1s, and nup2 $\Delta$ strains during a glucose shutdown time course. (Left panel) Short time course. (Right panel) A similar experiment including longer time points. 3C was performed using the primers indicated in A. (E) Mlp1 ChIP analysis on HXK1. (Left) Mlp1-GFP ChIP analysis on HXK1 at UAS, promoter, ORF, and 3'-end locations (as indicated in A) under overnight glucose (GLU) and galactose (GAL) conditions. The GAL1 UAS was a positive control (Luthra et al. 2007). Values are normalized to GLU conditions. (Right) Mlp1-GFP ChIP on HXK1 at $60 \mathrm{~min}$ and $4.5 \mathrm{~h}$ of glucose repression. Values are shown as immunoprecipitation/input after normalization to a non-GFP-tagged control strain. Values approaching 1 reflect background signals.

conditions (O'Sullivan et al. 2004; Ansari and Hampsey 2005; Singh and Hampsey 2007). Interestingly, however, when a glucose shutdown $3 \mathrm{C}$ time course was performed, we observed that the HXK1 gene loop was maintained for up to 60 min following transcription repression (Fig. 1D). However, as seen above, ChIP analysis showed Pol II 
leaving the $H X K 1$ promoter by $1 \mathrm{~min}$ of glucose shutdown, and it was completely absent from the entire gene by $5-8 \mathrm{~min}$ (Fig. 1C). This indicates that Pol II is not involved in maintaining the gene loop structure during glucose repression. Significantly, in an mlp1s strain (but not nup2A), this gene loop loses stability and the 3C signal is lost within $5 \mathrm{~min}$ of repression (Fig. 1D). This suggests that NPC-associated Mlp1 is required for maintenance of the gene loop during glucose repression.

\section{Mlp1 is associated with the $5^{\prime}$ and $3^{\prime}$ ends of HXK1}

To test this hypothesis, an Mlp1 ChIP was performed on HXK1 in wild-type cells. While Mlp1 was not detectable in internal regions of $H X K 1$, it was robustly detected at the $5^{\prime}$ UAS end and the $3^{\prime}$ end of the gene (Fig. 1E, left panel). These signals were transcription-dependent, as they were specific to galactose conditions. This $5^{\prime}-3^{\prime}$ bimodal distribution of Mlp1 is strongly indicative of its involvement in a gene loop structure.

Significantly, this $5^{\prime}-3^{\prime}$ distribution of Mlp1 was detected at $60 \mathrm{~min}$ after glucose repression, during which the gene loop is still maintained in wild-type cells, but not at $4.5 \mathrm{~h}$, when the loop is lost (Fig. 1D,E, right panels). These data strongly suggest that Mlp1 acts to maintain gene loop structure following transcription repression.

\section{Mlp1 is required for faster Pol II recruitment and transcriptional memory in HXK1}

To establish the function of these NPC-tethered gene loops, we next examined the connection between NPC association and gene reinduction in HXK1. Time-course analysis of mRNA expression and Pol II ChIP was performed under conditions of repeated glucose repression and galactose induction. Induction time courses were performed under three conditions as detailed in Figure 2A: (1) first-round induction after overnight culture growth in repressive glucose conditions (dotted lines); (2) second-round reinduction after a short 60-min intervening period of repression (solid line; these are referred to as memory conditions); and (3) second-round reinduction after a longer 4.5 -h intervening period of repression (dashed line).

Under reinduction conditions where memory of previous transcription may be retained due to only a short

(A)

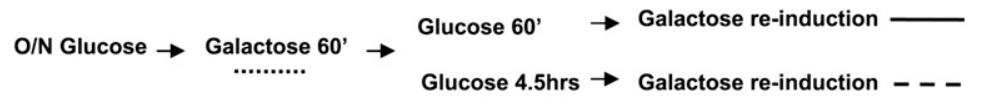

(B)

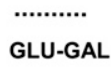

GLU-GAL-GLU60'-GAL
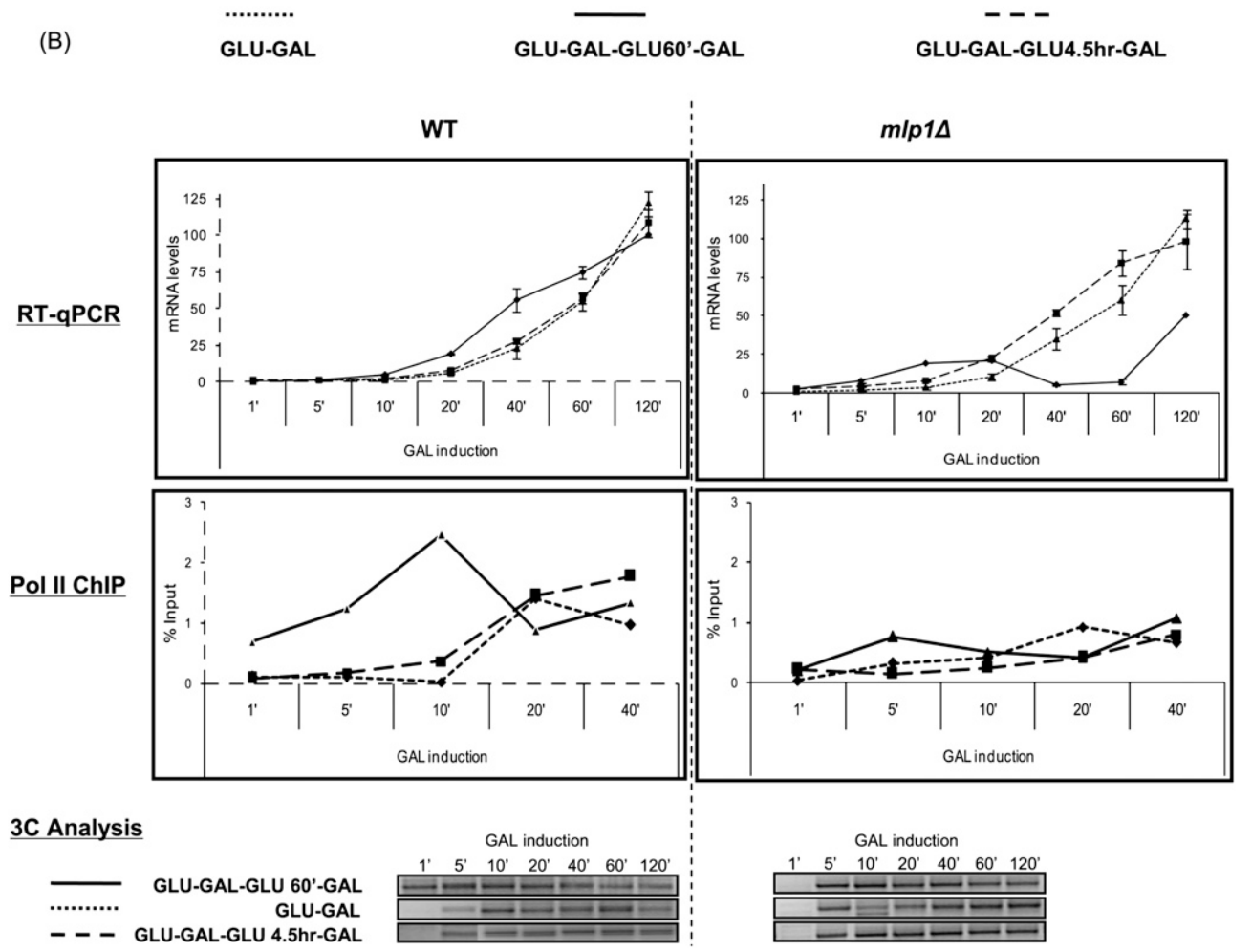

Figure 2. Mlp1-facilitated gene loops are required for transcriptional memory in HXK1. (A) Schematic of galactose induction and reinduction time course used for the following data sets. (B) HXK1 RT-qPCR (top panel), Pol II ChIP (middle panel), and 3C (bottom panel) data showing comparative kinetics between wild type (left panel) and mlp1s (right panel) under the culture conditions described in $A$. 3C primers are the same as in Figure 1A. The primer pair used for RT-qPCR as well as the ChIP probe is denoted " $\mathrm{C}^{\prime}$ in Figure 1A. 
60-min period of intervening glucose repression (Fig. 2B, solid line), we observe that $H X K 1$ shows a transcriptional memory effect - the rate of $H X K 1$ mRNA accumulation was doubled compared with longer-term repressed cells (Fig. 2B, RT-qPCR, left panel, $40 \mathrm{~min}$ ). Interestingly, we observe that this more rapid mRNA accumulation memory is regulated at the level of Pol II recruitment to the gene. As seen in Figure 2B (ChIP, left panel) there is a striking peak of Pol II occupancy of HXK1 at the preceding 10-min time point of the memory conditions, indicating that, upon reinduction, Pol II is recruited more rapidly to cells that display transcriptional memory.

In mlp1s mutants, however, transcriptional memory appears to be abolished, with the memory condition (Fig. 2B, solid line) lacking the faster mRNA reinduction effect (Fig. 2B, RT-qPCR, cf. left and right panels). We note that lack of Mlp1 actually causes a reduction in steady-state HXK1 mRNA levels in the memory conditions. As Mlp1 is implicated in mRNP surveillance at the NPC /Galy et al. 2004; Vinciguerra et al. 2005), this effect may be due to defects in mRNA export quality control under conditions in which the gene (and associated RNA) is still located close to the NPC. Furthermore, no increased Pol II levels were detectible for the mlp1s strain at the early 10-min time point, consistent with the lack of an mRNA accumulation memory. This further suggests a role for Mlp1 in faster Pol II recruitment during transcriptional memory conditions. Overall, these data imply an effect of Mlp1 on transcription memory-associated gene transcription.

\section{Mlp1-dependent gene loop presence is required} for HXK1 transcriptional memory

3C analyses of $H X K 1$ following galactose reinduction in a similar set of time-course experiments reveal that transcriptional memory is associated with gene loop retention during the intervening period of repression. As seen after a short 60-min intervening period of repression, gene loops are present at the very start of the subsequent memory reinduction conditions (Fig. 2B, analysis of 3C, top left panel). These results match our data above, in which we observed the continued maintenance of gene loops for up to $60 \mathrm{~min}$ of glucose repression (Fig. 1D). Combining the two sets of data, we predict that MGLs are maintained for up to $60 \mathrm{~min}$ of intervening repression, correlating with faster Pol II recruitment and reinduction if the gene is reinduced within this $60-\mathrm{min}$ period (memory conditions). Consistent with this model, we observed above that looping is eventually lost during longer-term 4.5-h and overnight repression conditions (Fig. 1D), and takes $5 \mathrm{~min}$ to reform following galactose induction (Fig. 2B, analysis of 3C, left panel). The lack of MGL presence at the beginning of these long-term induction/reinduction conditions matches the lack of faster Pol II recruitment.

Significantly, mlp1s cells were unable to maintain MGL structures for a 60-min period of intervening repression (loops were lost within $5 \mathrm{~min}$ in this strain) (Fig. 1D). Hence, we observe no MGL at the start of the predicted memory reinduction conditions (Fig. 2B, analysis of 3C, top right panel), thereby explaining the lack of faster Pol II recruitment observed above. Taken together, these data support a function for Mlp1-mediated gene loops in enhancing Pol II recruitment to inducible genes in transcriptional memory conditions.

\section{Mlp1-facilitated gene loop requirement for transcriptional memory extends to other inducible gene systems}

To determine the generality of our results, we extended our data on $H X K 1$ to a different inducible gene system. We used the large FMP27 gene, with its weak promoter replaced in genomic context by the regulatable GAL1 promoter (Strasser et al. 2002). This hybrid gene (GAL1::FMP27) is repressed by glucose and induced by galactose in a manner similar to $H X K 1$ (see Supplemental Fig. 1 for induction/repression time-course mRNA analysis). We demonstrated previously that GAL1::FMP27 forms a transcription-dependent gene loop based on 3C analysis (O'Sullivan et al. 2004). Additionally, previous studies have shown that similar GAL1 reporter genes localize to the NPC upon induction and are retained there for a period of time after transcriptional shutdown (Abruzzi et al. 2006; Chekanova et al. 2008; Vodala et al. 2008).

Using the same conditions of repeated glucose repression and galactose induction (Fig. 2A) to investigate transcriptional memory effects, we show that this gene system also displays accelerated mRNA expression levels and Pol II ChIP kinetics under memory conditions (Fig. 3B, left panel, solid line). An extended time-course analysis with time points taken at $3-8 \mathrm{~h}$ in the wild-type strain illustrates that stable steady-state mRNA levels increase greatly between 3 and $5 \mathrm{~h}$, with the memory conditions still providing the highest levels (Supplemental Fig. 4). A drop in mRNA levels is observed between 5 and $8 \mathrm{~h}$, indicating possible transcriptional autoregulation or mRNA stability regulation at these much later time points. Significantly, the transcriptional memory effect observed for wild type is eliminated in an mlp1s strain (Fig. 3B, cf. left and right panels). Furthermore, as with $H X K 1$, the GAL1::FMP27 MGL is maintained for up to $60 \mathrm{~min}$ of glucose repression in wild-type cells (Fig. 3B, analysis of 3C, left panel). Consequently, as the galactose induction 3C time course then shows, this MGL is present at the very beginning of reinduction memory conditions (Fig. 3B, solid line), thereby enabling faster Pol II recruitment and mRNA reinduction.

The GAL1::FMP27 gene loop, like that of $H X K 1$, is lost more rapidly during glucose repression in the mlp1s strain (Fig. 3B, analysis of $3 \mathrm{C}$ analysis, right panel). This results in the absence of an MGL at the start of galactose reinduction even under the predicted memory conditions, and consequently a lack of faster Pol II recruitment and mRNA accumulation effects. Loop formation does eventually occur upon active transcription, mirroring the kinetics observed under nonmemory conditions (Fig. 3B, right panel, dotted/dashed lines). 
(A)

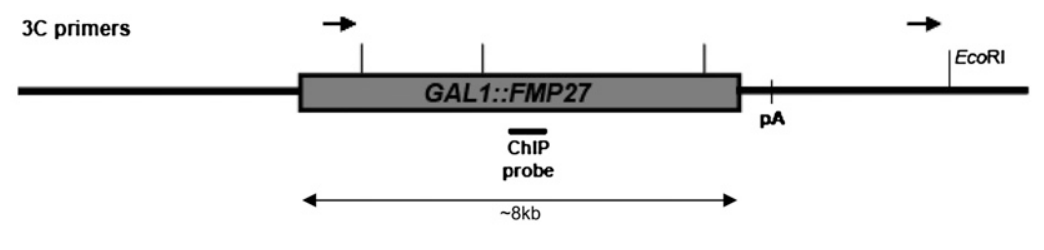

(B)

GLU-GAL

GLU-GAL-GLU60'-GAL

GLU-GAL-GLU4.5hr-GAL
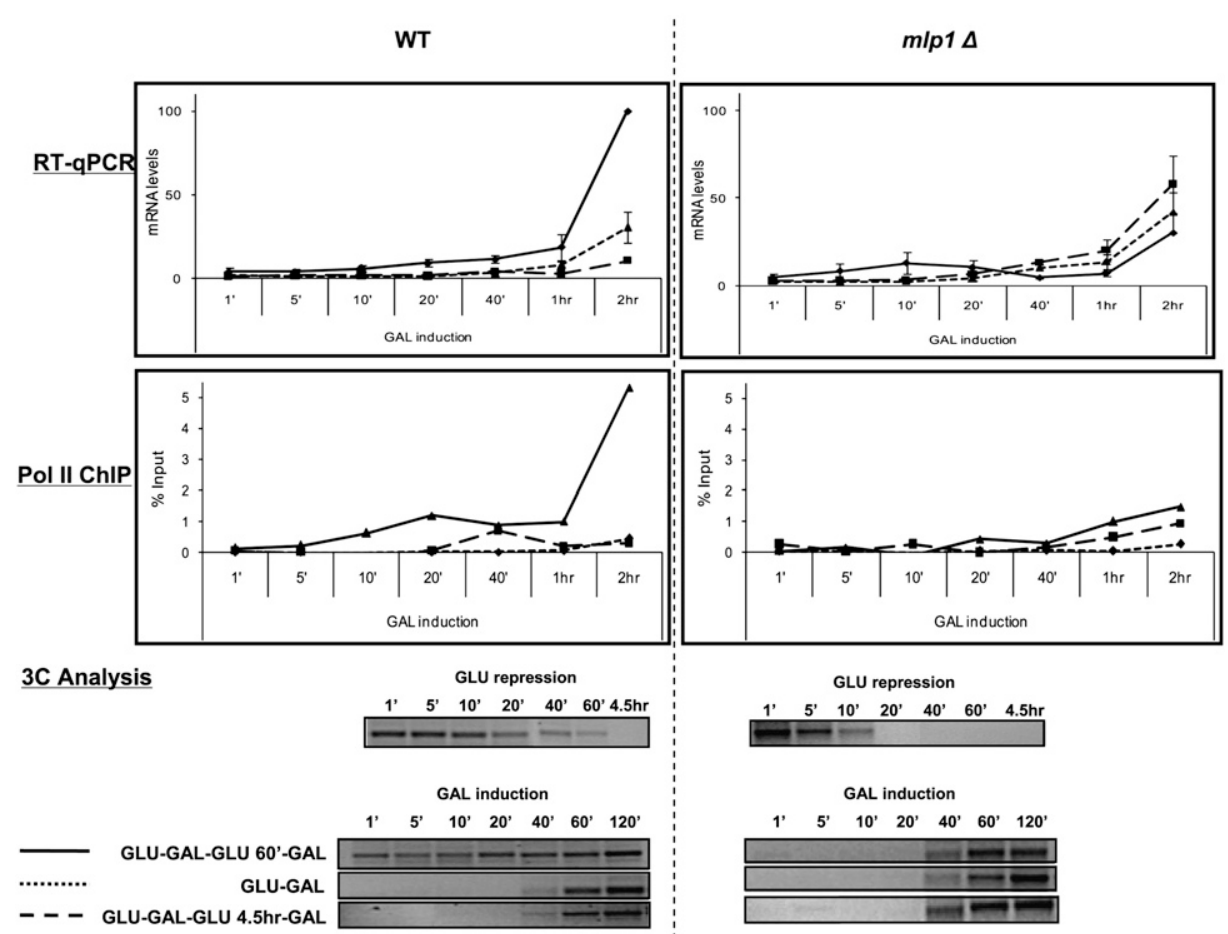

(C)
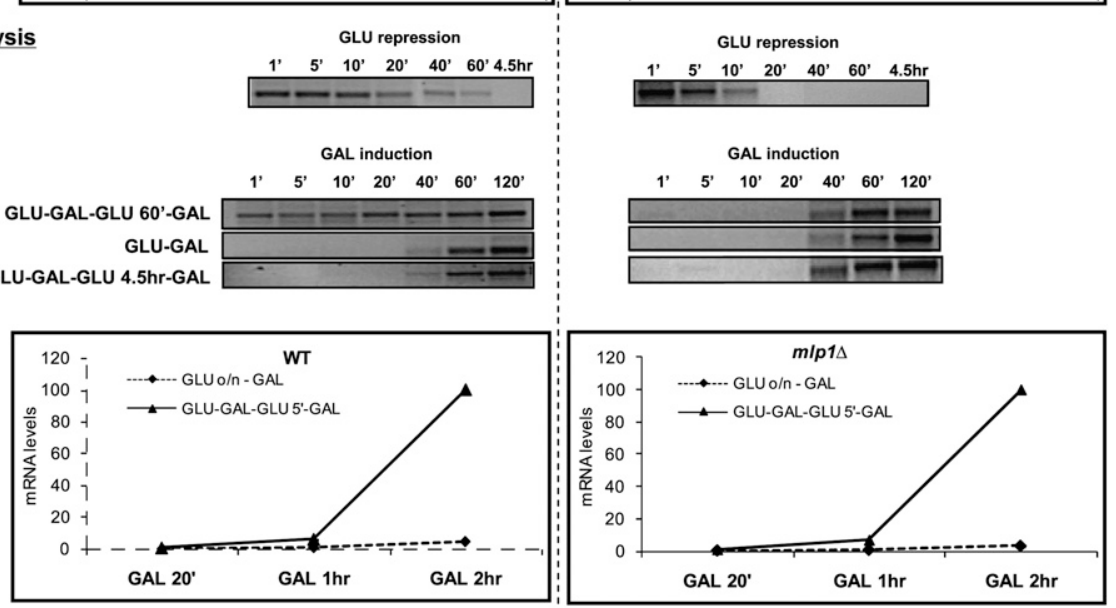

Figure 3. Mlp1-facilitated gene loops are required for fast transcriptional memory in GAL1::FMP27. (A) Schematic of GAL1::FMP27 indicating positions of $3 \mathrm{C}$ primers (above) and ChIP probe (below). Vertical lines depict EcoRI restriction sites used in 3C analysis. $(B)$ GAL1::FMP27 RT-qPCR (top panel), Pol II ChIP (middle panel), and 3C (bottom panel) data showing comparative kinetics between wild type (left panel) and mlp1s (right panel) under culture conditions described in Figure 2A. (C) GAL1::FMP27 RT-qPCR time course of wild-type and mlp1 $\Delta$ strains reinduced after a very short 5-min period of intervening repression (during which the MGL is still present, even in $m l p 1 \Delta)$. These data are an average of multiple biological repeats. Error bars have been included but are very small, so not clearly visible.

We note that the kinetics of GAL1::FMP27 induction are slower compared with those observed for HXK1, and attribute this effect to the greater length of this gene ( $\sim 8 \mathrm{~kb}$ for FMP27 vs. $\sim 1.5 \mathrm{~kb}$ for HXK1). Furthermore, it takes the GAL1::FMP27 gene loop 40 min to form upon transcriptional activation, a longer time than observed for HKX1 (5 min) (see Fig. 2B, analysis of $3 \mathrm{C}$ ). This again is likely to be due to its greater length. In addition to the length effect, it is also possible that the different promoter and 3 '-regulatory elements contained within these genes, as well as the heterologous nature of the GAL1::FMP27 hybrid gene, affects the rate of transcription and mRNA formation, thereby further contributing to the differing kinetics seen between these genes.

Taken together, the data from HXK1 and GAL1::FMP27 demonstrate that Mlp1 is required to maintain MGLs during intervening periods of repression. It is also required, together with Mlp1-facilitated MGLs, for the rapid transcriptional memory effects seen in these inducible genes. 
Gene loop presence is sufficient for transcriptional memory

In order to directly assess the loop requirement, we performed a further time-course analysis in which GAL1::FMP27 was reinduced within $5 \mathrm{~min}$ of glucose repression. As was seen in the $3 \mathrm{C}$ analysis in Figure 3B, the MGL loses stability and is maintained for only $\sim 10$ min in the mlp1s strain. Therefore, we determined whether memory would still be displayed if the gene was reinduced within this very short repression time during which the loop is still present in an mlp1s strain; i.e., within $5-10 \mathrm{~min}$. A time-course analysis equivalent to that in Figure $3 \mathrm{~B}$ was performed and reinduction was carried out after a very short 5-min intervening repression time period. It should be noted here that complete glucose repression was observed at the glucose 5-min time point at which cells were reinduced. As seen in Figure 3C, wild-type cells display the expected profile of a clear mRNA accumulation memory phenotype, completely consistent with the data in Figure 3B. Remarkably, however, mlp1s cells are now also able to display memory, mirroring the wild-type effect almost identically. These results show that the gene is indeed still able to display memory even in $m l p 1 \Delta$ cells, as long as it is reinduced at a time point at which the MGL is still present. These data also argue that the memory loss seen for $m l p 1 \Delta$ in Figure 3B is not an indirect effect caused by general NPC loss of integrity.

Taken together, our data indicate that while Mlp1 is needed for maintenance of MGLs during repression, causing loss of loop stability in an mlp1s strain, the gene is still able to display memory if reinduced before the complete loss of loop integrity. This strongly suggests that MGL presence is sufficient to enable faster transcription initiation memory.

\section{Mlp1 is required for HXK1 localization to the NPC}

Our data thus far indicate that genes maintain an Mlp1tethered MGL during short intervening periods of repression, thus giving rise to transcriptional memory effects. We next investigated the requirement of Mlp1 in HXK1 localization and tethering to the NPC. Mlp1 is an NPC protein, visibly located along the nuclear periphery ring (Feuerbach et al. 2002). In order to determine the localization of HXK1 relative to Mlp1 and the NPC, we used a strain that expresses a GFP-tagged hybrid form of another NPC protein, Nup49 (Nup49-GFP), to label the nuclear periphery. This strain also possesses an insertion of $2561 a c^{\text {op }}$ repeats upstream of $H X K 1$ together with expressed LacI-GFP to label the $l a c^{\mathrm{op}}-H X K 1$ gene locus (Taddei et al. 2006). Using fluorescent light microscopy, we visualized and categorized the LacI-GFP-HXK1 locus (visible as a green dot in Fig. 4A,B) relative to the nuclear focal plane zones I, II, and III (Fig. 4A,B). HXK1 loci stably tethered to the NPC would be located in the zone I crosssectional area of the nuclear focal plane. HXK1 is known to localize to the nuclear periphery upon induction, and in accordance with this, we observe a significantly higher percentage of cells with $H X K 1$ zone I localization during
(A)

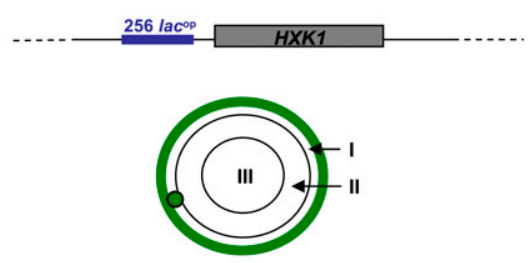

(B)

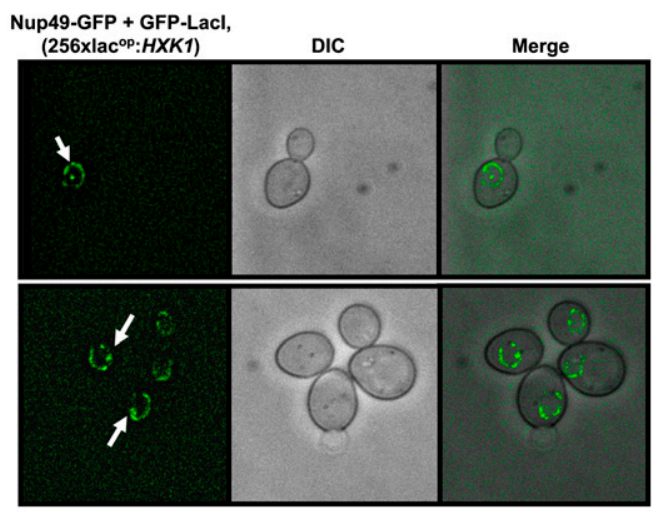

(C)

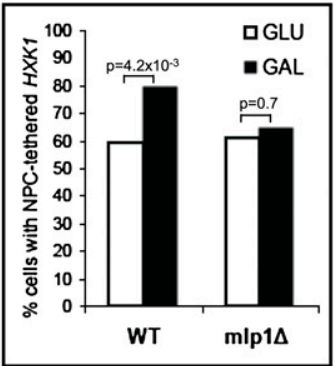

Figure 4. Mlp1 is required for HXK1 localization to the NPC upon activation. $(A, t o p)$ Schematic of yeast cells with 256 lac $^{\mathrm{op}}$ repeats upstream of HXK1 and expressing LacI-GFP and Nup49GFP fusions (Taddei et al. 2006). (Bottom) Schematic of the nuclear focal plane with the Nup49-GFP-labeled nuclear periphery and categorization of zones I, II, and III. (B) Representative microscopy analysis of the LacI-GFP-HXK1 dot in subnuclear positions of zone III (top) and zone I (bottom). (C) Percentage of cells with LacI-GFP-HXK1 tethered to the NPC upon galactose activation in wild type and $\operatorname{mlp} 1 \Delta$.

active galactose compared with repressive glucose conditions (Fig. 4C), consistent with previous observations (Taddei et al. 2006). Interestingly, however, an mlp1s strain shows no such induction-specific $H X K 1$ localization to the NPC. This underlines the essential role of Mlp1 in tethering $H X K 1$ to the NPC. Given that the Mlp1 protein is detected at the $5^{\prime}$ and $3^{\prime}$ ends of the HXK1 gene (Fig. 1E), and that an mlp1s strain shows a loss of MGLs and a lack of faster Pol II recruitment, these data reinforce our prediction that NPC-associated gene loop structures are required for the maintenance of transcriptional memory.

\section{Gene looping and transcriptional memory is disrupted in sua7-1 mutants}

We next investigated the direct requirement of gene loops for memory. We analyzed a mutant strain in which gene 
loop formation is specifically disrupted, but in which the NPC remains intact. The yeast TFIIB homolog Sua7 is involved in transcription initiation and start site selection, and a sua 7-1 mutant has been shown to specifically impair gene loop formation in a wide range of $S$. cerevisiae genes, while maintaining stable transcription at the permissive temperature (Pinto et al. 1992; Singh and Hampsey 2007). As seen in Figure 5A, a clear HXK1 gene loop is seen in an isogenic wild-type strain during active transcription and an MGL is maintained for up to $60 \mathrm{~min}$ of glucose repression. Loop formation, however, is impaired in a sua 7-1 mutant. An induction/repression timecourse analysis shows that, although an isogenic wild- type strain displays transcriptional memory as expected (Fig. 5B), the gene loop-disrupting sua 7-1 mutation causes a severe reduction in this memory effect (Fig. 5C). This supports a strong connection between the presence of gene loops and the maintenance of memory.

Furthermore, an Mlp1 ChIP shows that while $H X K 1$ associates with Mlp1 in a wild-type strain, both during active transcription and for up to $60 \mathrm{~min}$ of repression (consistent with data in Fig. 1E), a sua7-1 mutant loses this Mlp1 tether (Fig. 5D). As this is a strain in which the NPC components remain intact, the loss of the geneNPC tether is likely caused by the disruption of the gene loop. This signifies the important role of both gene loops
(A)

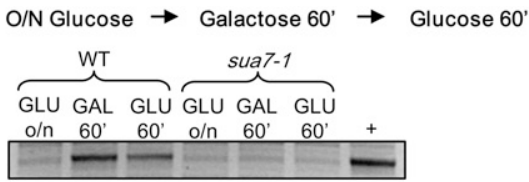

(B)

$\mathrm{O} / \mathrm{N}$ Glucose $\rightarrow$ Galactose induction

O/N Glucose $\rightarrow$ Galactose 60' $\rightarrow$ Glucose $60^{\prime} \rightarrow$ Galactose re-induction

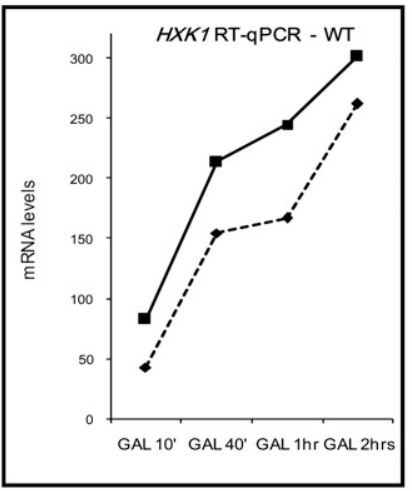

(C)

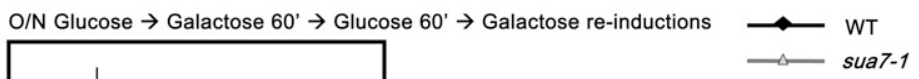

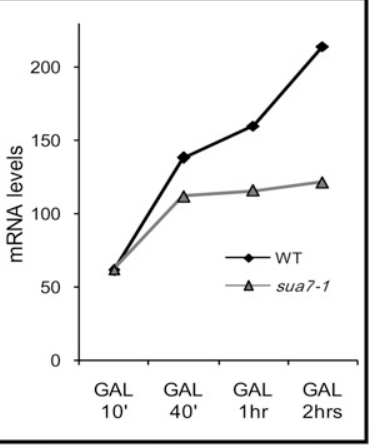

(D)

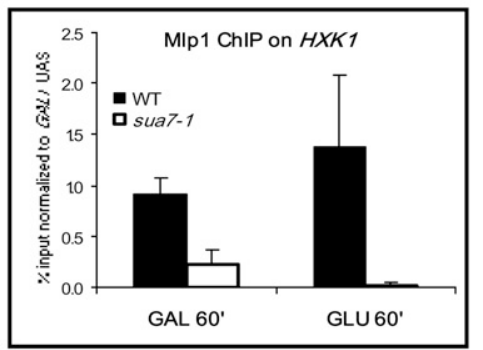

Figure 5. Disrupted gene looping in sua7-1 causes loss of NPC tethering and transcriptional memory. $(A)$ HXK1 3C gene loop analysis of sua7-1 and its isogenic wild type at time points of overnight glucose repression (GLU o/n), active galactose transcription (GAL $\left.60^{\prime}\right)$, and a subsequent 60 -min glucose repression (GLU 60'). The plus sign $(+)$ refers to the 3C PCRpositive control (see Supplemental Fig. 1C for details). $3 \mathrm{C}$ primers are the same as in Figure $1 \mathrm{~A} .(B$, top $)$ Schematic of galactose induction and reinduction time course used for the following RT-qPCR data sets. (Bottom) RT-qPCR data showing comparative naïve induction and second-round reinduction kinetics in wild type. $(C)$ RT-qPCR data showing comparative reinduction kinetics between wild type and sua7-1. (D) Mlp1 ChIP on HXK1 performed on GFPtagged Mlp1 in wild-type and sua7-1 strains during galactose induction and a subsequent 60 -min glucose repression (memory condition). 
Tan-Wong et al.

and their associated NPC tether in maintaining transcriptional memory.

\section{INO1 lacks repression memory loops and displays no transcriptional memory}

To strengthen the direct connection between gene loop function and transcriptional memory, as well as to eliminate the possibility that the observed gene loopmemory connection is an indirect effect through NPCassociated function, we finally investigated another inducible transcription system, INO1. This encodes the enzyme inositol-1-phosphate synthase, which catalyzes the conversion of glucose-6-phosphate to inositol-1-phosphate. INO1 transcription is repressed in the presence of inositol and is induced when grown in media lacking inositol. Similar to $H X K 1$ and GAL1 reporter constructs, INO1 also localizes to the nuclear periphery upon transcriptional activation and is retained there for a period after transcriptional repression (Brickner et al. 2007). As an inducible system comparable with that of $H X K 1$ and GAL1::FMP27, we were interested in determining whether INO1 also displayed similar gene loop profiles and transcriptional memory effects.

Consistent with all yeast and mammalian genes analyzed thus far, INO1 shows a clear gene loop between the promoter and 3' polyadenylation (pA) signal region during active transcription (Fig. 6C; Supplemental Fig. 2D). A repeated repression/induction time-course analysis was then performed under memory and nonmemory conditions as detailed in Figure 6B. Cells were repressed for an intervening short-term period of $1 \mathrm{~h}$ or a long-term period of $12 \mathrm{~h}$ before being reinduced. The memoryerasing long-term repression was conducted for $12 \mathrm{~h}$ due to previous studies showing that INO1 loses preferential nuclear peripheral localization and returns to random distribution within the nucleus only $12 \mathrm{~h}$ post-repression (Brickner et al. 2007). Interestingly, in contrast to HXK1
Figure 6. Absence of repression gene loop in INO1 correlates with lack of transcriptional memory. (A) Schematic of INO1 indicating positions of $3 \mathrm{C}$ primers. Vertical lines depict HaeIII restriction sites used in $3 \mathrm{C}$ analysis. $(B)$ Schematic of the $\pm 100 \mu \mathrm{M}$ inositol induction/repression time course used for $C$ and $D .(C)$ INO1 3C data showing the kinetics of INO1 loop presence under the culture conditions described in $B$. (3C primers) I2-I6 as depicted in $A$. The black arrow points to the $1-h$ period of intervening repression during which an MGL would predictably be retained. (D) INO1 RTqPCR data showing the kinetics of INO1 induction and reinduction under the culture conditions described in B. (E) Mlp1 ChIP on INO1 (on an Mlp1-GFP-tagged strain). The schematic at the top details the four time points at which the ChIPs were performed. Mlp1 ChIP at the GAL1 UAS was performed as a positive control for the ChIP (Luthra et al. 2007). As such, the time course was performed under differing glucose and galactose conditions (as indicated in the schematic) in order to ascertain Mlp1 presence on GAL1 UAS during active galactose conditions, and its absence during repressive glucose conditions.

(A) 3C primers

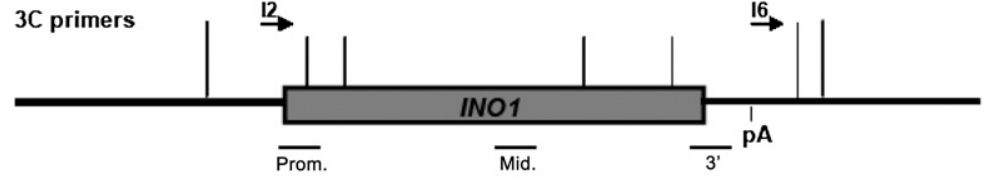

(B)

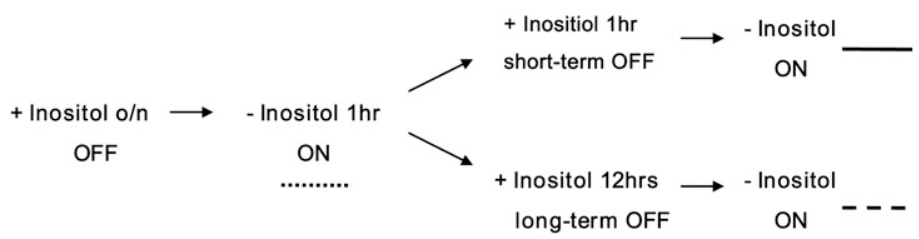

(C) $3 \mathrm{C}$

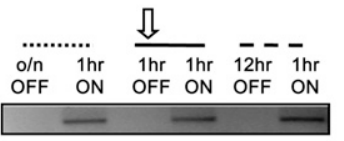

(D) INO1 RT-qPCR

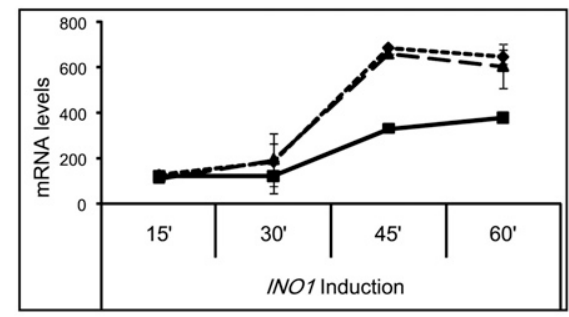

(E) Mlp1 ChIP on INO1
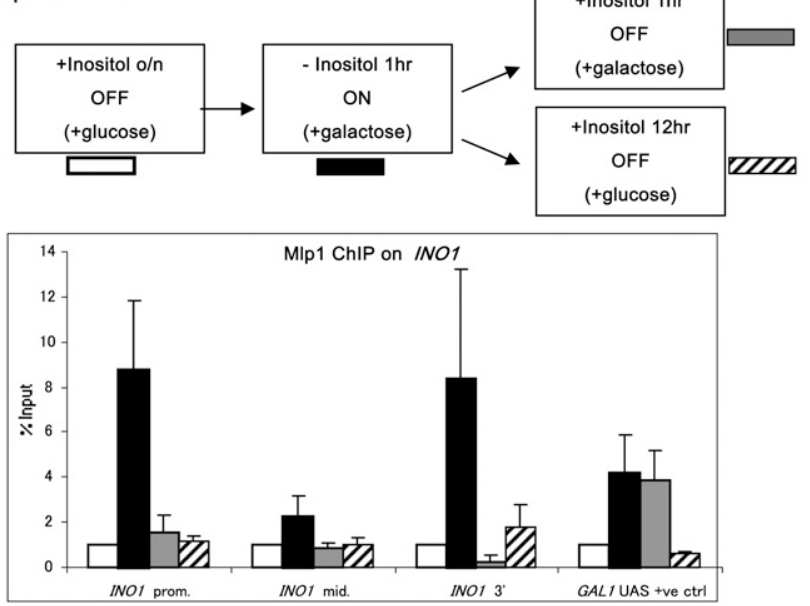
and GAL1::FMP27, INO1 does not maintain an MGL either during a short 1-h repression (Fig. 6C, solid line) or a longer 12-h repression (Fig. 6C, dashed line). Significantly, and as expected due to this lack of an MGL, we observe that INO1 transcription does not display a rapid mRNA accumulation reinduction memory (Fig. 6D). Indeed, the rate of INO1 mRNA accumulation is significantly reduced following reinduction after intervening short-term repression. Therefore, INO1 provides an example of a gene naturally lacking the maintenance of an MGL during repression, resulting in the absence of transcriptional memory.

These results showing a lack of INO1 memory are consistent with previous observations. Brickner et al. (2007) demonstrated that INO1 mRNA levels following second-round reinduction were significantly lower than initial induction levels (consistent with Fig. 6D). Also, these lower second-round reinduction mRNA levels were dependent on nuclear peripheral localization, since loss of NPC localization resulted in even lower mRNA levels. Interestingly, as seen in Figure 6E, Mlp1 is found associated with the INO1 promoter and $\mathrm{pA}$ regions during active transcription, but this Mlp1 association is lost during subsequent repression, even at a short-term repression time point of $1 \mathrm{~h}$. This is entirely consistent with the looping profile observed by 3C for INO1 in Figure 6C. These data are also consistent with the Mlp1 ChIP profiles seen above for sua 7-1, in which Mlp1 association was impaired in the sua 7-1 strain that lacked a gene loop. These data suggest that the absence of gene loops leads to the inability to maintain an Mlp1 tether, and together results in the lack of transcriptional memory effects.

\section{Discussion}

The results presented in this study provide a clear function for gene loop formation. We demonstrate that transcription-dependent gene loops in HXK1 and GAL1::FMP27 are maintained during intervening periods of repression, enabling faster Pol II recruitment and, thereby, faster mRNA accumulation upon reinduction. Mlp1, observed by ChIP at the base of the HXK1 5'-3' loop structure, is required for both this loop maintenance and for HXK1 tethering to the NPC, and consequently for transcriptional memory. Conversely, lack of gene looping in a sua7-1 mutant causes a loss of transcriptional memory, similar to INO1 that also lacks MGLs.

Our model (Fig. 7) proposes that when genes are fully active (i.e., in galactose conditions) they exist as a promoter- $3^{\prime}$-end gene loop structure. As formation of this loop upon initial induction does not require Mlp1 (Figs. $2 \mathrm{~B}, 3 \mathrm{~B}$, cf. wild type and mlp1s $3 \mathrm{C}$ upon initial induction), we predict that Pol II and/or other factors in the transcription apparatus may initially tether the gene loop (O'Sullivan et al. 2004). This actively transcribing looped gene then translocates to the nuclear periphery and is tethered to the NPC through Mlp1, which is required for the maintenance of MGLs during repression (Fig. 7B). This is consistent with previous studies showing the involvement of a gene's promoter and 3' untranslated region (UTR) in NPC localization (Abruzzi et al. 2006; Taddei et al. 2006). Long-term repression $(\geq 4.5 \mathrm{~h})$ results in the dismantling of the loop structure and a switch to a linear arrangement (as seen by 3C analysis) (Fig. 1D), with concurrent dissociation from the NPC (as shown by loss of Mlp1 association by ChIP after 4.5 h) (Fig. 1E). We propose that this dismantling of the NPC-associated gene loop scaffold leads to the loss of certain associated transcription factors. Reinduction requires the slow, de novo, stepwise recruitment of the transcriptional initiation complex and, subsequently, Pol II. In marked contrast, although short-term repression still results in the loss of Pol II (Fig. 1C), the gene retains its MGL interaction with the NPC through Mlp1 (Fig. 1D,E). We predict that transcription initiation factors are retained in this loop scaffold, providing a kinetically faster path to recruit Pol II and recommence transcription. This may well relate to the previously described reinitiation scaffold thought to enhance transcriptional initiation (Yudkovsky et al. 2000; Ansari and Hampsey 2005).

Consistent with this, a TBP ChIP performed on wildtype and mlp1s strains (Supplemental Fig. 7) shows that TBP is still abundantly present during a 1 -h glucose repression in a wild-type strain, but is significantly reduced in an mlp1s mutant strain in which memory is lost. This observation is highly consistent with previous data from other laboratories on other such transcription factors. The Gal4 transcriptional activator has been shown to be retained at the GAL1 UAS for a period of 1 $\mathrm{h}$ of glucose repression (Kundu et al. 2007). Furthermore, parallel work from the laboratory of Hampsey (Lainé et al. 2009) has also demonstrated that Gal4 is retained at the gene during glucose repression in a loop-dependent manner, being absent in conditions where there is no loop and no memory is observed. Taken together, these data strongly support the model outlined in Figure 7 in which transcription factors are retained in a scaffold for up to $1 \mathrm{~h}$ of repression in wild-type cells, thereby enabling faster reinduction within this period.

Remarkably, we note that despite a requirement for the Mlp1 tether in MGL maintenance during repression (Figs. $1 \mathrm{D}, 2 \mathrm{~B}, 3 \mathrm{~B})$, mlp1s cells are still able to display memory effects if reinduced prior to complete loop disintegration (Fig. 3C). This is a strong indication that MGLs are both necessary and sufficient for conferring transcriptional memory. As discussed above, the gene loop is likely held together by a transcription factor scaffold during active transcription and immediately post-transcription. Continued maintenance of MGL stability during repression, however, requires the Mlp1 tether.

Our model is further strengthened by the examples of the sua7-1 mutant and the INO1 gene. sua 7-1 impairs gene loop formation (but not transcription) at permissive temperatures and is therefore a useful control for specifically monitoring gene loop function and its negative effect on transcriptional memory maintenance. Consistent with our model above, we detect both a loss of transcriptional memory and a loss of HXK1 tethering to Mlp1 in sua 7-1 mutants (Fig. 5D). This is presumably due to the disruption of the gene loop and the inability of the 
Figure 7. Model for how Mlp1-facilitated gene loops function to retain transcriptional memory. (A) Elongation and active transcription during inductive growth conditions, with the gene tethered to the NPC in a loop confirmation. $(B)$ Repression for $\leq 60 \mathrm{~min}$ : The gene loop is retained at the NPC through Mlp1 involvement despite Pol II leaving the template. (C) Upon reinduction, Pol II is able to load onto the promoter and begin faster transcription faster due to retention of transcription factors in the loop scaffold. This causes the faster reinduction memory effects with consequent faster mRNA accumulation. $(D)$ With repression for $\geq 4.5 \mathrm{~h}$, gene loop conformation as well as the NPC association is eventually lost; certain transcription factors are released due to a lack of the loop scaffold. $(E)$ Upon reinduction, the transcription initiation complex forms de novo, resulting in comparatively slower kinetics of transcription reinduction. Gene loops reform upon transcription and the gene then becomes tethered to the NPC as in $A$ and $C$. Protein factors potentially involved in the loop scaffold are depicted in the schematic legend: Mex67 is known to be recruited to the promoter regions of GAL10 and HSP104 during gene activation and promotes gene anchoring through its association with Mlp1 (Dieppois et al. 2006). SAGA is recruited to the GAL UAS by Gal4 during galactose activation and SAGA complex proteins interact with Mlp1 at active genes, an interaction required for Mlp1 association with the GAL promoter (Luthra et al. 2007). SAGA-associated Sus1, a component of the mRNA export complex TREX-2, is necessary for nuclear peripheral positioning of active genes, and binds the NPC through Nup1 (Fischer et al. 2002; Rodriguez-Navarro et al. 2004; Cabal et al. 2006; Drubin et al. 2006; Chekanova et al. 2008). Nup2 interacts with promoters of active genes (Schmid et al. 2006).
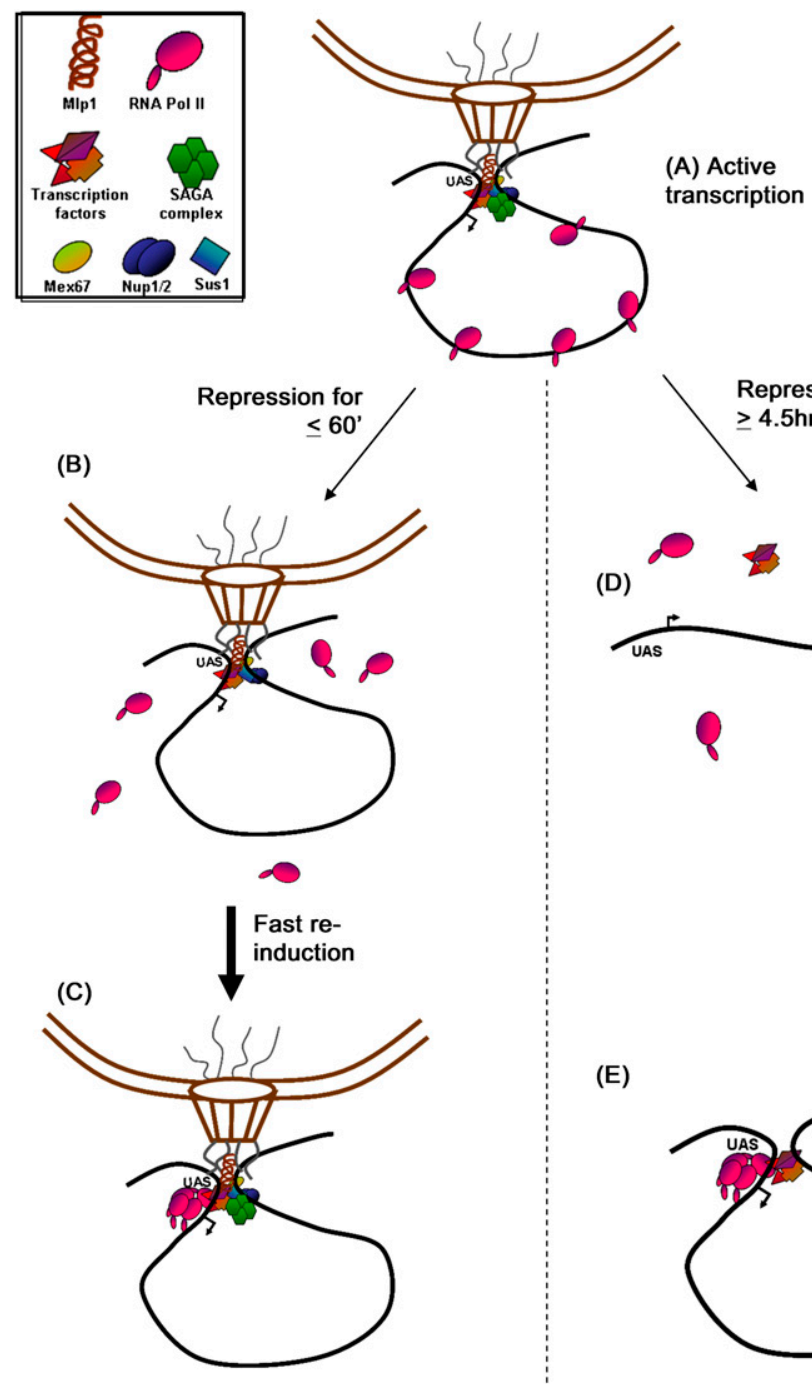

(D)

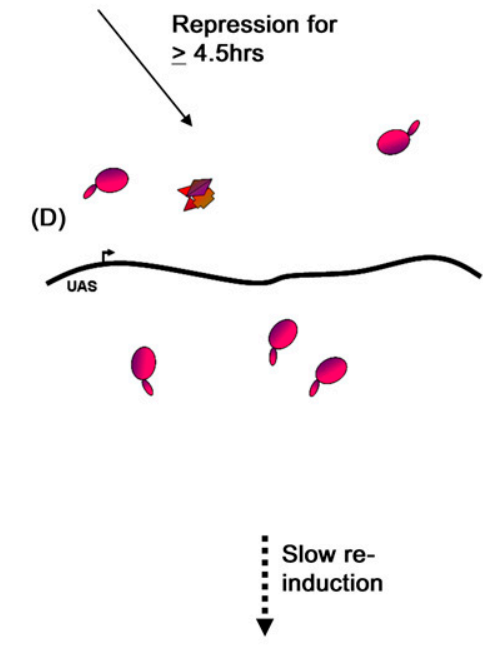

(E)

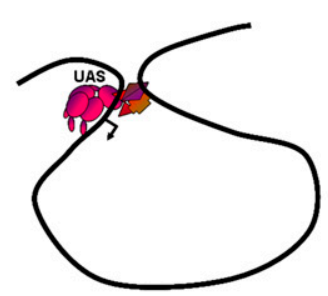

gene to hold in place an Mlp1-mediated loop scaffold. This supports the idea that MGLs and an Mlp1 tether to the NPC together function toward retaining transcriptional memory.

The results from INO1 reinforce the essential requirement of gene loops in maintaining transcriptional memory. Transcriptional memory is defined as a higher level of mRNA reinduction following short-term intervening repression (compared with after long-term repression). We confirm that INO1 displays no such faster reinduction memory. As seen in Figure 6D, second-round reinduction mRNA levels are, in effect, significantly lower than initial induction levels, similar to previous observations (Brickner et al. 2007). It was reported that these lower reinduction mRNA levels are dependent on nuclear peripheral localization, as loss of such localization results in even lower mRNA levels (Brickner et al. 2007).
Our studies observe that although INO1 is found associated with Mlp1 during active transcription, this Mlp1 association is lost during subsequent repression, even at a short-term repression time point of $1 \mathrm{~h}$ (Fig. 6E), consistent with the lack of an MGL at this time point. As INO1 has been reported previously to display nuclear peripheral positioning using fluorescence detection methods (Brickner et al. 2007), this suggests that the peripheral positioning during repression occurs in an Mlp1-independent manner and is separate from the memory-preserving looping pathway. Nup2 is the protein implicated by Brickner et al. (2007) in INO1 retention at the periphery, and our studies showed consistently that Nup2 deletion has no effect on MGL maintenance or on transcriptional memory effects (Fig. 1; Supplemental Fig. 5). These data therefore suggest that MGLs are specific to Mlp1, and that it is specifically Mlp1-mediated MGLs 
maintained during repression that function to retain transcriptional memory. Genes like INO1 that do not show Mlp1 association or Mlp1-mediated MGLs during repression do not display transcriptional memory effects.

In relation to INO1, we note that it is a gene that naturally lacks the maintenance of a repression MGL, and thereby does not display transcriptional memory. In biochemical terms, this is understandable, as INO1 may not require immediate rapid reinduction kinetics and may not have evolved to maintain an MGL. INO1 codes for inositol-1-phosphate synthase, which catalyzes the conversion of glucose-6-phosphate to inositol-1-phosphate, allowing the production of inositol (after a further step of inositol-1-phosphate dephosphorylation). In a switch to inositol-lacking (INO1-inductive) media after a short intervening period in inositol-containing (INO1-repressive) media, it is likely that immediate and rapid production of inositol-1-phosphate synthase (allowing for further production of inositol) would be unnecessary, since high inositol levels are likely still present in the cell. Furthermore, the biochemistry of phosphatidic acid metabolism may also help explain the differing INO1 transcription profiles. As discussed above, we as well as others (Brickner et al. 2007) observed that INO1 reinduction levels after short-term repression are lower than after long-term repression. INO1 transcription is regulated by phosphatidic acid, which is converted to phosphatidylinositol by the action of inositol in the media as well as by the Inol enzyme. It is therefore possible that the requirement of phosphatidic acid reappearance for INO1 activation may cause the slower reinduction profile observed (Brickner et al. 2007).

The results presented in this study can be viewed in light of studies that point to a role for RNA in gene-NPC anchoring. A genome-wide ChIP analysis showed that Mlp1 association with active genes is RNA-dependent and is enriched at the $3^{\prime}$ end of the genes (Casolari et al. 2005). Furthermore, post-transcriptional retention of RNA and their adjacent DNA loci dots at the nuclear periphery is dependent on the nuclear exosome components Rrp6 and Lrp1, suggesting RNA involvement in gene tethering to the nuclear periphery (Abruzzi et al. 2006; Vodala et al. 2008). It is also instructive to relate our results to previously reported mechanisms for transcription memory in yeast. Both specific histone variants (H2A.Z) and chromatin remodeling enzymes (SWI/SNF) are implicated in this process (Brickner et al. 2007; Kundu et al. 2007), and may well act on the proposed MGL-NPC complex that we observe during short-term repression. However, we show that loss of gene looping, either by deletion of Mlp1 or by a sua 7-1 mutation, is sufficient to erase transcriptional memory. This argues against redundant mechanisms of transcriptional memory and, rather, suggests a synergistic role, possibly facilitated through the MGL scaffold and mediated by Mlp1. Interestingly, NPC-associated Nup2 has also been implicated previously in recruiting inducible genes to the nuclear periphery and in tethering chromatin boundary elements (Ishii et al. 2002; Schmid et al. 2006; Brickner et al. 2007). However, Nup2 deletion had no effect on
MGL maintenance (Fig. 1D) or on transcriptional memory in these studies (Supplemental Fig. 5).

Also to be considered is the study by Tzamarias and colleagues (Zacharioudakis et al. 2007), who demonstrated a cytoplasmic role of Gall-GFP in providing transcriptional memory. This Gall effect could well influence the gene loop structure through direct contact across the NPC. However, we show that while there is a loss of memory in mlp1s mutants (Figs. 2, 3), this is not due to a differential effect of the Gall, since no detectible Gall-GFP is present in either wild type or mlp1s during the memory time points performed in these studies. Thus, we show by Western blot (Supplemental Fig. 8A) and FACS analysis (Supplemental Fig. 8B) that Gall is absent in both wild type and mlp1s, even during the periods of intervening repression during which wild-type cells retain memory but mlp1s cells do not. We presume that this is due to the relatively short prior galactose induction period $(1 \mathrm{~h})$ during which time Gall has not accumulated. Consequently, the memory effects seen here are specific to Mlp1 and its associated gene loops but are independent of a cytoplasmic Gall influence. Indeed, it remains likely that the MGL effect we observed reflects a short-term process, while the Gall memory effect may become significant when longer-term galactose induction is employed, resulting in Gall accumulation.

Finally, it is instructive to consider potential parallels between our work in yeast and that of higher eukaryotes. It is interesting to note that, like in yeast, there is evidence of active chromatin presence at the nuclear periphery in higher eukaryotes as well. DNaseI-sensitive chromatin, an indicator of transcription potential, was found to localize preferably at the nuclear periphery in vertebrates (Hutchison and Weintraub 1985). Furthermore, dosage compensation in Drosophila melanogaster by male-specific lethal (MSL) protein-mediated transcriptional up-regulation of the male $\mathrm{X}$ chromosome also has links to the nuclear periphery. Knockdown of nuclear pore proteins triggers a loss of MSL localization to the $\mathrm{X}$ chromosome and of male dosage compensation, indicating that MSLmediated recruitment of the male X-chromosomal genes to the nuclear pore is essential for transcriptional upregulation (Mendjan et al. 2006). Therefore, while gene loop function parallels cannot yet be drawn, as transcriptional memory is yet to be described in higher eukaryotes, this does remain a distinct possibility.

Overall, we demonstrate here a key regulatory function for intragenic loops, and show that gene loops, as ascribed to a now substantial number of actively transcribed genes, are likely to play an important role in efficient gene expression.

\section{Materials and methods}

Yeast strains

HXK1 and GAL1::FMP27 experiments were performed with

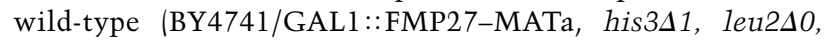

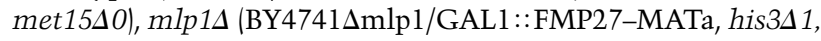

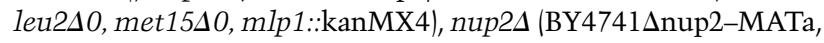

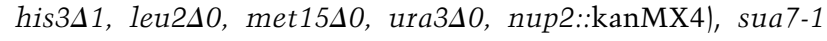


(YMH124/YDW546), and the sua7-1 isogenic wild-type (YMH14/T16) strains (Pinto et al. 1992; Singh and Hampsey 2007). INO1 experiments were also performed with the above BY4741 wild-type strain.

Mlp1-TAP ChIPs were performed on YSC1178 TAP-tagged MLP1 (with endogenous promoter; Open Biosystems), using the

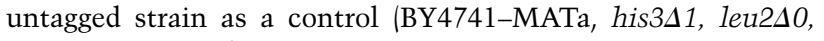
met15 0 , ura3 $\Delta 0$ ).

Mlp1-GFP ChIPs were performed on the sua7-1 and isogenic wild-type strains, where Mlp1 was GFP-tagged in-frame at the end of the $M L P 1$ coding region using homologous recombination and GFP-KanMX6 selection. GFP-tagged constructs were checked by Western blot analysis (Supplemental Fig. 9).

Microscopy analysis was performed on a strain tagged with $256 l a c^{\text {op }}$ repeats upstream of HXK1 on chromosome 6 and expressing Nup49-GFP and LacI-GFP (kind gift from Susan Gasser) (Hediger et al. 2002; Taddei et al. 2006). The corresponding mlp1s strain was made using an mlp1::kanMX6 deletion by homologous recombination.

Gall Western blots and FACS analysis (for Supplemental Fig. 8) were performed with strains BY4741 and BY4741 $\Delta \mathrm{mlp1}$, which were GAL1-GFP-tagged by inserting GFP-KanMX6 inframe at the $3^{\prime}$ end of the GAL1 coding region.

All strains were cultured at $30^{\circ} \mathrm{C}$, except for the sua $7-1$ and its isogenic wild-type strains (and their Mlp1-GFP-tagged derivatives), which were cultured at a permissive temperature of $25^{\circ} \mathrm{C}$.

\section{Induction/repression time courses}

For experiments with HXK1 and GAL1::FMP27, cultures were grown overnight in $\mathrm{YP}+2 \%$ glucose media and induction/ repression/reinduction was performed as detailed in the figures. For glucose-to-galactose shifts, cells were spun down and washed prior to resuspension in $\mathrm{YP}+2 \%$ galactose media. For galactoseto-glucose shifts, $4 \%$ glucose was added to existing media. Samples were taken simultaneously for RNA and ChIP analysis at the specified time points. Cultures were handled in a $30^{\circ} \mathrm{C}$ room to avoid minor fluctuations due to temperature shifts, especially for the very quick time courses.

For experiments with INO1, strains were grown overnight in SC-inositol media (SC-URA-Inositol, Formedium DSCK212 supplemented with $76 \mathrm{mg} / \mathrm{mL}$ uracil), with $100 \mu \mathrm{M}$ myo-inositol. INO1 was activated by washing cells and resuspending in SCinositol media, and INO1 was repressed by addition of $100 \mu \mathrm{M}$ myo-inositol. All cultures were continuously diluted and maintained at logarithmic phase of growth.

\section{RNA analysis}

RNA isolation was performed using hot phenol and RNA was DNase-treated prior to oligodT reverse transcription. qPCR was performed on a Corbett Rotorgene 3000 or 6000 machine using 72 reaction plates. Experiments were carried out in triplicate on reverse-transcribed samples and non-reverse-transcribed controls in all experiments, and were quantitated using Rotorgene 3000 software using standard curves on every plate. All RTqPCR values were normalized to their respective $A D H 1$ controls at each time point and are shown as a ratio of a standard internal control time-point value (this internal control time point was repeated on every qPCR plate in order to ensure accurate and standard normalization and comparison between qPCR plates and across an entire set of RNA time points). Further controls were performed using another control transcript, PYK1, to confirm that $A D H 1$ levels remain stable during glucose/galactose treatments and between wild-type and sua 7-1 strains, and can be used safely as an RNA normalization control in our experimental conditions (Supplemental Fig. 6). All primer sequences are listed in Supplemental Table 1.

\section{ChIP analysis}

Pol II ChIPs were performed following published protocol (O'Sullivan et al. 2004) using the Rpb1(Y80) antibody (Santa Cruz Biotechnologies, sc-25758). qPCR was carried out in triplicate for all ChIP experiments and quantitated using Rotorgene 3000 software using a standard curve on every plate. ADH1 ChIP controls were performed for all samples, and all percent input values for HXK1 and GAL1:FMP27 were normalized to their respective $A D H 1$ ChIP controls at every time point and are shown as a ratio of a standard internal control value. All ChIP profiles were reproducible over multiple (three or more) independent biological repeats.

Mlp1-TAP ChIPs on HXK1 were performed using a TAPtagged Mlpl strain following published protocol (Keogh and Buratowski 2004). Values are shown as immunoprecipitation/ input after normalization to the untagged control strain.

Mlp1-GFP ChIPs on HXK1 were performed using the anti-GFP (FL) antibody (Santa Cruz Biotechnologies, sc-8334). ChIP qPCRs were performed simultaneously on the GAL1 UAS locus as a positive control for the Mlp1 ChIP, and percent input values on HXK1 were normalized to their respective GAL1 UAS control values.

TBP ChIPs were performed using a monoclonal anti-TBP antibody (Sigma, catalog no. T1827) on the wild-type and mlp1s strains. TBP was detected by qPCR at the GAL1::FMP27 UAS.

\section{$3 C$ analysis}

$3 \mathrm{C}$ was performed using the restriction enzymes MseI for HXK1, EcoRI for GAL1::FMP27, and HaeIII for INO1 following published 3C protocol (O'Sullivan et al. 2004). Primers used for the 3C PCRs in each instance are indicated in the relevant figure. Multiple positive and negative controls were performed for all $3 \mathrm{C}$ analyses on each gene and are shown in Supplemental Figure 2A-D.

\section{Microscopy}

Live cells (grown overnight in glucose or galactose) were imaged using a Metamorph-driven Zeiss Axioplan 2 light microscope. DIC (100-msec exposure) and GFP (4000-msec exposure) Z-stack analysis was performed using six to 11 image stacks of $0.2-\mu \mathrm{m}$ step size. Two-dimensional deconvolution followed by subsequent GFP dot analysis was performed using the Metamoprh software. Quantitative analysis of GFP dot position was performed following published protocol (Hediger et al. 2002). In brief, HXK1-lacI-GFP position was determined by measuring the GFP dot to nuclear periphery distance $(\mathrm{x})$ and the nuclear diameter $(\mathrm{y})$, and categorizing the $\mathrm{x} / \mathrm{y}$ value into zone $\mathrm{I}(0.184 \times$ nuclear radius r), zone II (0.184r-0.422r), or zone III (0.422r), as in Figure 4A. The percentage of NPC-tethered cells in zone I was then calculated with $n=65-112$ cells for each strain and condition. Nonmobile LacI-GFP-HXK1 dots located in zone I were scored as localized and tethered to the NPC. Statistical analysis was performed using a $\chi^{2}$ test comparing zone I percentages and $P$-values obtained using $1^{\circ}$ of freedom. Statistical significance was determined using a 95\% confidence interval. Glucose/galactose response RNA controls were simultaneously performed on the cultures used for microscopy (data not shown).

\section{Western blot analysis}

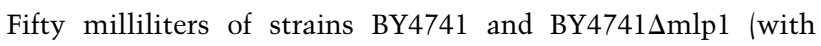
GAL1-GFP) were collected at log phase. Whole-cell extracts 
were prepared using glass beads in extraction buffer $(200 \mathrm{mM}$ Tris at $\mathrm{pH} 8.0,150 \mathrm{mM} \mathrm{NH}_{4} \mathrm{SO}_{4}, 10 \%$ glycerol, $1 \mathrm{mM}$ EDTA, 2 $\mathrm{mM}$ DTT, protease inhibitors). Protein concentration was measured using a nanodrop spectrophotometer, and $200 \mu \mathrm{g}$ of protein (per sample) were run on an $8 \%$ SDS-PAGE gel. Blocking was done for $1 \mathrm{~h}$ in TBST $+5 \%$ instant dried milk, primary antibody incubations were done at 1:250 dilutions for $1 \mathrm{~h}$ (anti-actin, Sigma, A2066; anti-GFP [FL], Santa Cruz Biotechnologies, sc8334), and secondary antibody incubations were also done for $1 \mathrm{~h}$ (1:5000 dilution; anti-rabbit IgG peroxidase, Sigma, A9169).

\section{FACS analysis}

Galactose activation experiments were performed with cells grown in $\mathrm{YP}+2 \%$ glucose and were kept at $\mathrm{OD}_{600}<0.2$ by continuous dilution. After 16-20 h growth, cells were washed with water and activated in YP $+2 \%$ galactose for $1 \mathrm{~h}$, and subsequently were repressed by $4 \%$ glucose addition. Samples were taken at 1 and $4.5 \mathrm{~h}$ of glucose repression. Gall-GFP-tagged strains grown in $\mathrm{YP}+2 \%$ galactose overnight were used as positive controls, and untagged strains were used as negative controls. For FACS analysis, $200 \mu \mathrm{L}$ of the cultures were harvested, washed, and resuspended in PBS. FACS analysis was performed with a Calibur instrument (Becton Dickinson) with 30,000 cells.

\section{Acknowledgments}

We are grateful to Ana Rondon for initial discussion on this project, and to her and Chris Norbury for advice on the manuscript. We also thank Hannah Mischo for assistance with the FACS analysis, Alan Waimann and Mike Shaw for assistance with the microscopy, and Dr. Marriot of the Statistical Consultancy Service at the University of Oxford Department of Statistics. This work was supported by a Wellcome Trust program grant to N.J.P. H.W. was supported by an ORS scholarship and a University of Oxford, Lincoln College Keith Murray Scholarship.

\section{Note added in proof}

Parallel studies from the laboratory of M. Hampsey (Lainé et al. 2009) are also published in this issue. Their results also demonstrate that gene loops confer transcriptional memory on the GAL10 and SEN1 genes.

\section{References}

Abruzzi KC, Belostotsky DA, Chekanova JA, Dower K, Rosbash M. 2006. 3'-End formation signals modulate the association of genes with the nuclear periphery as well as mRNP dot formation. EMBO J 25: 4253-4262.

Ansari A, Hampsey M. 2005. A role for the CPF 3'-end processing machinery in RNAP II-dependent gene looping. Genes \& Dev 19: 2969-2978.

Brickner JH, Walter P. 2004. Gene recruitment of the activated INO1 locus to the nuclear membrane. PLOS Biol 2: e342. doi: 10.1371/journal.pbio.0020342.

Brickner DG, Cajigas I, Fondufe-Mittendorf Y, Ahmed S, Lee PC, Widom J, Brickner JH. 2007. H2A.Z-mediated localization of genes at the nuclear periphery confers epigenetic memory of previous transcriptional state. PLoS Biol 5: e81. doi: 10.1371/ journal.pbio.0050081.

Cabal GG, Genovesio A, Rodriguez-Navarro S, Zimmer C, Gadal O, Lesne A, Buc H, Feuerbach-Fournier F, Olivo-Marin JC, Hurt EC, et al. 2006. SAGA interacting factors confine sub-diffusion of transcribed genes to the nuclear envelope. Nature 441: 770-773.

Casolari JM, Brown CR, Komili S, West J, Hieronymus H, Silver PA. 2004. Genome-wide localization of the nuclear transport machinery couples transcriptional status and nuclear organization. Cell 117: 427-439.

Casolari JM, Brown CR, Drubin DA, Rando OJ, Silver PA. 2005. Developmentally induced changes in transcriptional program alter spatial organization across chromosomes. Genes \& Dev 19: 1188-1198.

Chekanova JA, Abruzzi KC, Rosbash M, Belostotsky DA. 2008. Sus1, Sac3, and Thp1 mediate post-transcriptional tethering of active genes to the nuclear rim as well as to non-nascent mRNP. RNA 14: 66-77.

Dieppois G, Iglesias N, Stutz F. 2006. Cotranscriptional recruitment to the mRNA export receptor Mex67p contributes to nuclear pore anchoring of activated genes. Mol Cell Biol 26: $7858-7870$.

Drubin DA, Garakani AM, Silver PA. 2006. Motion as a phenotype: The use of live-cell imaging and machine visual screening to characterize transcription-dependent chromosome dynamics. BMC Cell Biol 7: 19. doi: 10.1186/14712121-7-19.

Feuerbach F, Galy V, Trelles-Sticken E, Fromont-Racine M, Jacquier A, Gilson E, Olivo-Marin JC, Scherthan H, Nehrbass U. 2002. Nuclear architecture and spatial positioning help establish transcriptional states of telomeres in yeast. Nat Cell Biol 4: 214-221.

Fischer T, Strasser K, Racz A, Rodriguez-Navarro S, Oppizzi M, Ihrig P, Lechner J, Hurt E. 2002. The mRNA export machinery requires the novel Sac3p-Thplp complex to dock at the nucleoplasmic entrance of the nuclear pores. $E M B O J$ 21: 5843-5852.

Galy V, Gadal O, Fromont-Racine M, Romano A, Jacquier A, Nehrbass U. 2004. Nuclear retention of unspliced mRNAs in yeast is mediated by perinuclear Mlp1. Cell 116: 63-73.

Hediger F, Neumann FR, Van Houwe G, Dubrana K, Gasser SM. 2002. Live imaging of telomeres: $y \mathrm{Ku}$ and Sir proteins define redundant telomere-anchoring pathways in yeast. Curr Biol 12: $2076-2089$.

Herrero P, Galindez J, Ruiz N, Martinez-Campa C, Moreno F. 1995. Transcriptional regulation of the Saccharomyces cerevisiae HXK1, HXK2 and GLK1 genes. Yeast 11: 137-144.

Hutchison N, Weintraub H. 1985. Localization of DNAase I-sensitive sequences to specific regions of interphase nuclei. Cell 43: 471-482.

Ishii K, Arib G, Lin C, Van Houwe G, Laemmli UK. 2002. Chromatin boundaries in budding yeast: The nuclear pore connection. Cell 109: 551-562.

Keogh MC, Buratowski S. 2004. Using chromatin immunoprecipitation to map cotranscriptional mRNA processing in Saccharomyces cerevisiae. Methods Mol Biol 257: 1-16.

Kundu S, Horn PJ, Peterson CL. 2007. SWI/SNF is required for transcriptional memory at the yeast GAL gene cluster. Genes \& Dev 21: 997-1004.

Lainé J-P, Singh BN, Krishnamurthy S, Hampsey, M. 2009. A physiological role for gene loops in yeast. Genes \& Dev (this issue). doi: 10.1101/gad.1823609.

Luthra R, Kerr SC, Harreman MT, Apponi LH, Fasken MB, Ramineni S, Chaurasia S, Valentini SR, Corbett AH. 2007. Actively transcribed GAL genes can be physically linked to the nuclear pore by the SAGA chromatin modifying complex. J Biol Chem 282: 3042-3049.

Mason PB, Struhl K. 2005. Distinction and relationship between elongation rate and processivity of RNA polymerase II in vivo. Mol Cell 17: 831-840. 
Tan-Wong et al.

Mendjan S, Taipale M, Kind J, Holz H, Gebhardt P, Schelder M, Vermeulen M, Buscaino A, Duncan K, Mueller J, et al. 2006. Nuclear pore components are involved in the transcriptional regulation of dosage compensation in Drosophila. Mol Cell 21: 811-823.

O'Sullivan JM, Tan-Wong SM, Morillon A, Lee B, Coles J, Mellor J, Proudfoot NJ. 2004. Gene loops juxtapose promoters and terminators in yeast. Nat Genet 36: 1014-1018.

Perkins KJ, Lusic M, Mitar I, Giacca M, Proudfoot NJ. 2008. Transcription-dependent gene looping of the HIV-1 provirus is dictated by recognition of pre-mRNA processing signals. Mol Cell 29: 56-68.

Pinto I, Ware DE, Hampsey M. 1992. The yeast SUA7 gene encodes a homolog of human transcription factor TFIIB and is required for normal start site selection in vivo. Cell 68: 977-988.

Rodriguez-Navarro S, Fischer T, Luo MJ, Antunez O, Brettschneider S, Lechner J, Perez-Ortin JE, Reed R, Hurt E. 2004. Sus1, a functional component of the SAGA histone acetylase complex and the nuclear pore-associated mRNA export machinery. Cell 116: $75-86$.

Sarma NJ, Haley TM, Barbara KE, Buford TD, Willis KA, Santangelo GM. 2007. Glucose-responsive regulators of gene expression in Saccharomyces cerevisiae function at the nuclear periphery via a reverse recruitment mechanism. Genetics 175: 1127-1135.

Schmid M, Arib G, Laemmli C, Nishikawa J, Durussel T, Laemmli UK. 2006. Nup-PI: The nucleopore-promoter interaction of genes in yeast. Mol Cell 21: 379-391.

Singh BN, Hampsey M. 2007. A transcription-independent role for TFIIB in gene looping. Mol Cell 27: 806-816.

Strasser K, Masuda S, Mason P, Pfannstiel J, Oppizzi M, Rodriguez-Navarro S, Rondon AG, Aguilera A, Struhl K, Reed R, et al. 2002. TREX is a conserved complex coupling transcription with messenger RNA export. Nature 417: 304308.

Taddei A, Van Houwe G, Hediger F, Kalck V, Cubizolles F, Schober H, Gasser SM. 2006. Nuclear pore association confers optimal expression levels for an inducible yeast gene. Nature 441: 774-778.

Tan-Wong SM, French JD, Proudfoot NJ, Brown MA. 2008. Dynamic interactions between the promoter and terminator regions of the mammalian BRCA1 gene. Proc Natl Acad Sci 105: 5160-5165.

Vinciguerra P, Iglesias N, Camblong J, Zenklusen D, Stutz F. 2005. Perinuclear Mlp proteins downregulate gene expression in response to a defect in mRNA export. $E M B O J$ 24: 813-823.

Vodala S, Abruzzi KC, Rosbash M. 2008. The nuclear exosome and adenylation regulate posttranscriptional tethering of yeast GAL genes to the nuclear periphery. Mol Cell 31: 104-113.

Yudkovsky N, Ranish JA, Hahn S. 2000. A transcription reinitiation intermediate that is stabilized by activator. Nature 408: 225-229.

Zacharioudakis I, Gligoris T, Tzamarias D. 2007. A yeast catabolic enzyme controls transcriptional memory. Curr Biol 17: 2041-2046. 


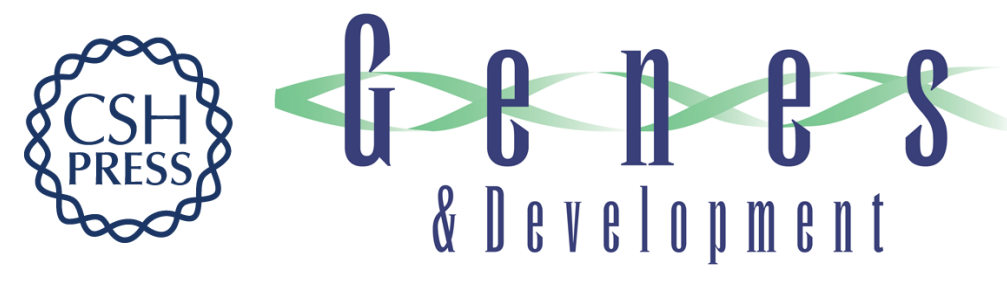

\section{Gene loops function to maintain transcriptional memory through interaction with the nuclear pore complex}

Sue Mei Tan-Wong, Hashanthi D. Wijayatilake and Nick J. Proudfoot

Genes Dev. 2009, 23:

Access the most recent version at doi:10.1101/gad.1823209

Supplemental
Material http://genesdev.cshlp.org/content/suppl/2009/11/19/23.22.2610.DC1

References This article cites 37 articles, 9 of which can be accessed free at:

http://genesdev.cshlp.org/content/23/22/2610.full.html\#ref-list-1

License Freely available online through the Genes \& Development Open Access option.

Email Alerting Receive free email alerts when new articles cite this article - sign up in the box at the top

Service right corner of the article or click here.

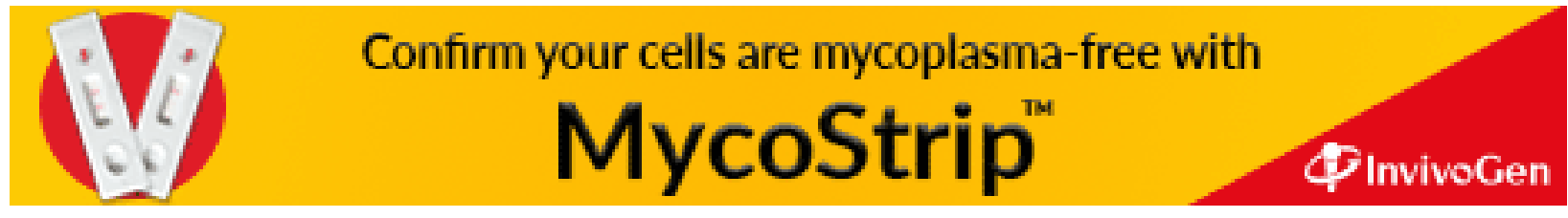

